\title{
Research Article \\ Cyclic Iterative Method for Strictly Pseudononspreading in Hilbert Space
}

\section{Bin-Chao Deng, Tong Chen, and Zhi-Fang Li}

School of Management, Tianjin University, Tianjin 300072, China

Correspondence should be addressed to Bin-Chao Deng,dbchao1985@yahoo.com.cn

Received 17 April 2012; Revised 1 June 2012; Accepted 1 June 2012

Academic Editor: Hong-Kun Xu

Copyright (C) 2012 Bin-Chao Deng et al. This is an open access article distributed under the Creative Commons Attribution License, which permits unrestricted use, distribution, and reproduction in any medium, provided the original work is properly cited.

Let $\left\{T_{i}\right\}_{i=1}^{N}$ be $N$ strictly pseudononspreading mappings defined on closed convex subset $C$ of a real Hilbert space $H$. Consider the problem of finding a common fixed point of these mappings and introduce cyclic algorithms based on general viscosity iteration method for solving this problem. We will prove the strong convergence of these cyclic algorithm. Moreover, the common fixed point is the solution of the variational inequality $\left\langle(\gamma f-\mu B) x^{*}, v-x^{*}\right\rangle \leq 0, \forall v \in \bigcap_{i=1}^{N} F_{i x}\left(T_{i}\right)$.

\section{Introduction}

Throughout this paper, we always assume that $C$ is a nonempty, closed, and convex subset of a real Hilbert space $H$. Let $B: C \rightarrow H$ be a nonlinear mapping. Recall the following definitions.

Definition 1.1. $B$ is said to be

(i) monotone if

$$
\langle B x-B y, x-y\rangle \geq 0, \quad \forall x, y \in C,
$$

(ii) strongly monotone if there exists a constant $\alpha>0$ such that

$$
\langle B x-B y, x-y\rangle \geq \alpha\|x-y\|^{2}, \quad \forall x, y \in C,
$$

for such a case, $B$ is said to be $\alpha$-strongly-monotone, 
(iii) inverse-strongly monotone if there exists a constant $\alpha>0$ such that

$$
\langle B x-B y, x-y\rangle \geq \alpha\|B x-B y\|^{2}, \quad \forall x, y \in C,
$$

for such a case, $B$ is said to be $\alpha$-inverse-strongly monotone,

(iv) $k$-Lipschitz continuous if there exists a constant $k \geq 0$ such that

$$
\|B x-B y\| \leq k\|x-y\| \quad \forall x, y \in C .
$$

Remark 1.2. Let $F=\mu B-\gamma f$, where $B$ is a $k$-Lipschitz and $\eta$-strongly monotone operator on $H$ with $k>0$ and $f$ is a Lipschitz mapping on $H$ with coefficient $L>0,0<\gamma \leq \mu \eta / L$. It is a simple matter to see that the operator $F$ is $(\mu \eta-\gamma L)$-strongly monotone over $H$, that is:

$$
\langle F x-F y, x-y\rangle \geq(\mu \eta-\gamma L)\|x-y\|^{2}, \quad \forall(x, y) \in H \times H .
$$

Following the terminology of Browder-Petryshyn [1], we say that a mapping $T$ : $D(T) \subseteq H \rightarrow H$ is

(1) $k$-strict pseudocontraction if there exists $k \in[0,1)$ such that

$$
\|T x-T y\|^{2} \leq\|x-y\|^{2}+k\|x-T x-(y-T y)\|^{2}, \quad \forall x, y \in D(T)
$$

(2) $k$-strictly pseudononspreading if there exists $k \in[0,1)$ such that

$$
\|T x-T y\|^{2} \leq\|x-y\|^{2}+k\|x-T x-(y-T y)\|^{2}+2\langle x-T x, y-T y\rangle,
$$

for all $x, y \in D(T)$,

(3) nonspreading in [2] if

$$
\|T x-T y\|^{2} \leq\|T x-y\|^{2}+\|T y-x\|^{2}, \quad \forall x, y \in C .
$$

It is shown in [3] that (1.8) is equivalent to

$$
\|T x-T y\|^{2} \leq\|x-y\|^{2}+2\langle x-T x, y-T y\rangle, \quad \forall x, y \in C
$$

Clearly every nonspreading mapping is 0-strictly pseudononspreading. Iterative methods for strictly pseudononspreading mapping have been extensively investigated; see $[2,4-6]$.

Let $C$ be a closed convex subset of $H$, and let $\left\{T_{i}\right\}_{i=1}^{N}$ be $n k_{i}$-strictly pseudocontractive mappings on $C$ such that $\bigcap_{i=1}^{N} F_{i x}\left(T_{i}\right) \neq \emptyset$. Let $x_{1} \in C$ and $\left\{\alpha_{n}\right\}_{n=1}^{\infty}$ be a sequence in $(0,1)$. In [7], Acedo and $\mathrm{Xu}$ introduced an explicit iteration scheme called the followting cyclic algorithm 
for iterative approximation of common fixed points of $\left\{T_{i}\right\}_{i=1}^{N}$ in Hilbert spaces. They define the sequence $\left\{x_{n}\right\}$ cyclically by

$$
\begin{gathered}
x_{1}=\alpha_{0} x_{0}+\left(1-\alpha_{0}\right) T_{0} x_{0} ; \\
x_{2}=\alpha_{1} x_{1}+\left(1-\alpha_{1}\right) T_{1} x_{1} ; \\
\vdots \\
x_{N}=\alpha_{N-1} x_{N-1}+\left(1-\alpha_{N-1}\right) T_{N-1} x_{N-1} ; \\
x_{N+1}=\alpha_{N} x_{N}+\left(1-\alpha_{N}\right) T_{0} x_{N} ; \\
\vdots
\end{gathered}
$$

In a more compact form, they rewrite $x_{n+1}$ as

$$
x_{N+1}=\alpha_{N} x_{N}+\left(1-\alpha_{N}\right) T_{N} x_{n}
$$

where $T_{N}=T_{i}$, with $i=n(\bmod N), 0 \leq i \leq N-1$. Using the cyclic algorithm (1.12), Acedo and $\mathrm{Xu}$ [7] show that this cyclic algorithm (1.12) is weakly convergent if the sequence $\left\{\alpha_{n}\right\}$ of parameters is appropriately chosen.

Motivated and inspired by Acedo and $\mathrm{Xu}$ [7], we consider the following cyclic algorithm for finding a common element of the set of solutions of $k_{i}$-strictly pseudononspreading mappings $\left\{T_{i}\right\}_{i=1}^{N}$. The sequence $\left\{x_{n}\right\}_{i=1}^{\infty}$ generated from an arbitrary $x_{1} \in H$ as follows:

$$
\begin{gathered}
x_{1}=\alpha_{0} \gamma f\left(x_{0}\right)+\left(I-\mu \alpha_{0} B\right) T_{\omega_{0}} x_{0} ; \\
x_{2}=\alpha_{1} \gamma f\left(x_{1}\right)+\left(I-\mu \alpha_{1} B\right) T_{\omega_{1}} x_{1} ; \\
\vdots \\
x_{N}=\alpha_{N-1} \gamma f\left(x_{N-1}\right)+\left(I-\mu \alpha_{N-1} B\right) T_{\omega_{N-1}} x_{N-1} ; \\
x_{N+1}=\alpha_{N} \gamma f\left(x_{N}\right)+\left(I-\mu \alpha_{N} B\right) T_{\omega_{0}} x_{N} ; \\
\vdots
\end{gathered}
$$

Indeed, the algorithm above can be rewritten as

$$
x_{n+1}=\alpha_{n} \gamma f\left(x_{n}\right)+\left(I-\mu \alpha_{n} B\right) T_{\omega_{[n]}} x_{n}
$$

where $T_{\omega_{[n]}}=\left(I-\omega_{[n]}\right) I+\omega_{[n]} T_{[n]}, T_{[n]}=T_{n} \bmod N$; namely, $T_{[N]}$ is one of $T_{1}, T_{2}, \ldots, T_{N}$ circularly. 


\section{Preliminaries}

Throughout this paper, we write $x_{n} \rightarrow x$ to indicate that the sequence $\left\{x_{n}\right\}$ converges weakly to $x . x_{n} \rightarrow x$ implies that $\left\{x_{n}\right\}$ converges strongly to $x$. The following definitions and lemmas are useful for main results.

Definition 2.1. A mapping $T$ is said to be demiclosed, if for any sequence $\left\{x_{n}\right\}$ which weakly converges to $y$, and if the sequence $\left\{T x_{n}\right\}$ strongly converges to $z$, then $T(y)=z$.

Definition 2.2. $T: H \rightarrow H$ is called demicontractive on $H$, if there exists a constant $\alpha<1$ such that

$$
\|T x-q\|^{2} \leq\|x-q\|^{2}+\alpha\|x-T x\|^{2}, \quad \forall(x, q) \in H \times F_{i x}(T) .
$$

Remark 2.3. Every $k$-strictly pseudononspreading mapping with a nonempty fixed point set $F_{i x}(T)$ is demicontractive (see $\left.[8,9]\right)$.

Remark 2.4 (See [10]). Let $T$ be a $\alpha$-demicontractive mapping on $H$ with $F_{i x}(T) \neq \emptyset$ and $T_{\omega}=$ $(1-\omega) I+\omega T$ for $\omega \in(0, \infty)$ :

(A1) $T \alpha$-demicontractive is equivalent to

$$
\langle x-T x, x-q\rangle \geq \frac{1}{2}(1-\alpha)\|x-T x\|^{2}, \quad \forall(x, q) \in H \times F_{i x}(T),
$$

(A2) $F_{i x}(T)=F_{i x}\left(T_{\omega}\right)$ if $\omega \neq 0$.

Remark 2.5. According to $I-T_{\omega}=\omega(I-T)$ with $T$ being a $k$-strictly pseudononspreading mapping, we obtain

$$
\left\langle x-T_{\omega} x, x-q\right\rangle \geq \frac{\omega(1-k)}{2}\|x-T x\|^{2}, \quad \forall(x, q) \in H \times F_{i x}(T) .
$$

Proposition 2.6 (see [2]). Let $C$ be a nonempty closed convex subset of a real Hilbert space $H$, and let $T: C \rightarrow C$ be a $k$-strictly pseudononspreading mapping. If $F_{i x}(T) \neq \emptyset$, then it is closed and convex.

Proposition 2.7 (see [2]). Let $C$ be a nonempty closed convex subset of a real Hilbert space $H$, and let $T: C \rightarrow C$ be a k-strictly pseudononspreading mapping. Then $(I-T)$ is demiclosed at 0 .

Lemma 2.8 (see [11]). Let $\left\{\tau_{n}\right\}$ be a sequence of real numbers that does not decrease at infinity, in the sense that there exists a subsequence $\left\{\tau_{n_{j}}\right\}_{j \geq 0}$ of $\left\{\tau_{n}\right\}$ which satisfies $\tau_{n_{j}}<\tau_{n_{j}+1}$ for all $j \geq 0$. Also consider the sequence of integers $\{\delta(n)\}_{n \geq n_{0}}$ defined by

$$
\delta(n)=\max \left\{k \leq n \mid \tau_{k}<\tau_{k+1}\right\}
$$

Then $\{\delta(n)\}_{n \geq n_{0}}$ is a nondecreasing sequence verifying $\lim _{n \rightarrow \infty} \delta(n)=\infty, \forall n \geq n_{0}$; it holds that $\tau_{\delta(n)}<\tau_{\delta(n)+1}$ and we have

$$
\tau_{n}<\tau_{\delta(n)+1}
$$


Lemma 2.9. Let $H$ be a real Hilbert space. The following expressions hold:

(i) $\|t x+(1-t) y\|^{2}=t\|x\|^{2}+(1-t)\|y\|^{2}-t(1-t)\|x-y\|^{2}, \quad \forall x, y \in H, \forall t \in[0,1]$,

(ii) $\|x+y\|^{2} \leq\|x\|^{2}+2\langle y, x+y\rangle, \quad \forall x, y \in H$.

Lemma 2.10 (see [6]). Let $C$ be a closed convex subset of a Hilbert space $H$, and let $T: H \rightarrow H$ be a $k$-strictly pseudononspreading mapping with a nonempty fixed point set. Let $k \leq \omega<1$ be fixed and define $T_{\omega} C \rightarrow C$ by

$$
T_{\omega}(x)=(1-\omega)(x)+\omega T(x), \quad \forall x \in C .
$$

Then $F_{i x}\left(T_{\omega}\right)=F_{i x}(T)$.

Lemma 2.11. Assume $C$ is a closed convex subset of a Hilbert space $H$.

(a) Given an integer $N \geq 1$, assume, $T_{i}: H \rightarrow H$ is a $k_{i}$-strictly pseudononspreading mapping for some $k_{i} \in[0,1),(i=1,2, \ldots, N)$. Let $\left\{\lambda_{i}\right\}_{i=1}^{N}$ be a positive sequence such that $\sum_{i=1}^{N} \lambda_{i}=1$. Suppose that $\left\{T_{i}\right\}_{i=1}^{N}$ has a common fixed point and $\bigcap_{i=1}^{N} F_{i x}\left(T_{i}\right) \neq \emptyset$. Then,

$$
F_{i x}\left(\sum_{i=1}^{N} \lambda_{i} T_{i}\right)=\bigcap_{i=1}^{N} F_{i x}\left(T_{i}\right) .
$$

(b) Assuming $T_{i}: H \rightarrow H$ is a $k_{i}$-strictly pseudononspreading mapping for some $k_{i} \in[0,1)$, $(i=1,2, \ldots, N)$, let $T_{\omega_{i}}=\left(1-\omega_{i}\right) I+\omega_{i} T_{i}, 1 \leq i \leq N$. If $\bigcap_{i=1}^{N} F_{i x}\left(T_{i}\right) \neq \emptyset$, then

$$
F_{i x}\left(T_{\omega_{1}} T_{\omega_{2}} \cdots T_{\omega_{N}}\right)=\bigcap_{i=1}^{N} F_{i x}\left(T_{\omega_{i}}\right)
$$

Proof. To prove (a), we can assume $N=2$. It suffices to prove that $F_{i x}(F) \subset F_{i x}\left(T_{1}\right) \cap F_{i x}\left(T_{2}\right)$, where $F=(1-\lambda) T_{1}+\lambda T_{2}$ with $0<\lambda<1$. Let $x \in F_{i x}(F)$ and write $V_{1}=I-T_{1}$ and $V_{2}=I-T_{2}$.

From Lemma 2.9 and taking $z \in F_{i x}\left(T_{1}\right) \cap F_{i x}\left(T_{2}\right)$ to deduce that

$$
\begin{aligned}
\|z-x\|^{2}= & \left\|(1-\lambda)\left(z-T_{1} x\right)+\lambda\left(z-T_{2} x\right)\right\|^{2} \\
= & (1-\lambda)\left\|z-T_{1} x\right\|^{2}+\lambda\left\|z-T_{2} x\right\|^{2}-\lambda(1-\lambda)\left\|T_{1} x-T_{2} x\right\|^{2} \\
\leq & (1-\lambda)\left(\|z-x\|^{2}+k\left\|x-T_{1} x\right\|^{2}\right)+\lambda\left(\|z-x\|^{2}+k\left\|x-T_{2} x\right\|^{2}\right) \\
& -\lambda(1-\lambda)\left\|T_{1} x-T_{2} x\right\|^{2} \\
= & \|z-x\|^{2}+k\left[(1-\lambda)\left\|V_{1} x\right\|^{2}+\lambda\left\|V_{2} x\right\|^{2}\right]-\lambda(1-\lambda)\left\|V_{1} x-V_{2} x\right\|^{2},
\end{aligned}
$$

it follows that

$$
\lambda(1-\lambda)\left\|V_{1} x-V_{2} x\right\|^{2} \leq k\left[(1-\lambda)\left\|V_{1} x\right\|^{2}+\lambda\left\|V_{2} x\right\|^{2}\right] .
$$


Since $(1-\lambda) V_{1} x+\lambda V_{2} x=0$, we obtain

$$
(1-\lambda)\left\|V_{1} x\right\|^{2}+\lambda\left\|V_{2} x\right\|^{2}=\lambda(1-\lambda)\left\|V_{1} x-V_{2} x\right\|^{2} .
$$

This together with (2.10) implies that

$$
(1-k) \lambda(1-\lambda)\left\|V_{1} x-V_{2} x\right\|^{2} \leq 0 .
$$

Since $0<\lambda<1$ and $k<1$, we get $\left\|V_{1} x-V_{2} x\right\|=0$ which implies $T_{1} x=T_{2} x$ which in turn implies that $T_{1} x=T_{2} x=x$ since $(1-\lambda) T_{1} x+\lambda T_{2} x=x$. Thus, $x \in F_{i x}\left(T_{1}\right) \cap F_{i x}\left(T_{2}\right)$.

By induction, we also claim that $F_{i x}\left(\sum_{i=1}^{N} \lambda_{i} T_{i}\right)=\bigcap_{i=1}^{N} F_{i x}\left(T_{i}\right)$ with $\left\{\lambda_{i}\right\}_{i=1}^{N}$ is a positive sequence such that $\sum_{i=1}^{N} \lambda_{i}=1,(i=1,2, \ldots, N)$.

To prove (b), we can assume $N=2$. Set $T_{\omega_{1}}=\left(1-\omega_{1}\right) I+\omega_{1} T_{1}$ and $T_{\omega_{2}}=\left(1-\omega_{2}\right) I+\omega_{1} T_{2}$, $0<k_{i}<\omega_{i}<1 / 2, i=1,2$. Obviously

$$
F_{i x}\left(T_{\omega_{1}}\right) \cap F_{i x}\left(T_{\omega_{2}}\right) \subset F_{i x}\left(T_{\omega_{1}} T_{\omega_{2}}\right) .
$$

Now we prove

$$
F_{i x}\left(T_{\omega_{1}}\right) \cap F_{i x}\left(T_{\omega_{2}}\right) \supset F_{i x}\left(T_{\omega_{1}} T_{\omega_{2}}\right)
$$

for all $q \in F_{i x}\left(T_{\omega_{1}} T_{\omega_{2}}\right)$ and $T_{\omega_{1}} T_{\omega_{2}} q=q$. If $T_{\omega_{2}} q=q$, then $T_{\omega_{1}} q=q$; the conclusion holds. From Lemma 2.10, we can know that $F_{i x}\left(T_{\omega_{1}}\right) \cap F_{i x}\left(T_{\omega_{2}}\right)=F_{i x}\left(T_{1}\right) \cap F_{i x}\left(T_{2}\right) \neq \emptyset$. Taking $p \in$ $F_{i x}\left(T_{\omega_{1}}\right) \cap F_{i x}\left(T_{\omega_{2}}\right)$, then

$$
\begin{aligned}
\|p-q\|^{2}= & \left\|p-T_{\omega_{1}} T_{\omega_{2}} q\right\|^{2}=\left\|p-\left[\left(1-\omega_{1}\right)\left(T_{\omega_{2}} q\right)+\omega_{1} T_{1} T_{\omega_{2}} q\right]\right\|^{2} \\
= & \left\|\left(1-\omega_{1}\right)\left(p-T_{\omega_{2}} q\right)+\omega_{1}\left(p-T_{1} T_{\omega_{2}} q\right)\right\|^{2} \\
= & \left(1-\omega_{1}\right)\left\|p-T_{\omega_{2}} q\right\|^{2}+\omega_{1}\left\|p-T_{1} T_{\omega_{2}} q\right\|^{2}-\omega_{1}\left(1-\omega_{1}\right)\left\|T_{\omega_{2}} q-T_{1} T_{\omega_{2}} q\right\|^{2} \\
\leq & \left(1-\omega_{1}\right)\left\|p-T_{\omega_{2}} q\right\|^{2}-\omega_{1}\left(1-\omega_{1}\right)\left\|T_{\omega_{2}} q-T_{1} T_{\omega_{2}} q\right\|^{2} \\
& +\omega_{1}\left[\left\|p-T_{\omega_{2}} q\right\|^{2}+k_{1}\left\|T_{\omega_{2}} q-T_{1} T_{\omega_{2}} q\right\|^{2}+2\left\langle p-T_{1} T_{\omega_{2}} p, T_{\omega_{2}} q-T_{1} T_{\omega_{2}} q\right\rangle\right] \\
= & \left(1-\omega_{1}\right)\left\|p-T_{\omega_{2}} q\right\|^{2}-\omega_{1}\left(1-\omega_{1}\right)\left\|T_{\omega_{2}} q-T_{1} T_{\omega_{2}} q\right\|^{2} \\
& +\omega_{1}\left[\left\|p-T_{\omega_{2}} q\right\|^{2}+k_{1}\left\|T_{\omega_{2}} q-T_{1} T_{\omega_{2}} q\right\|^{2}\right] \\
\leq & \left\|p-T_{\omega_{2}} q\right\|^{2}-\omega_{1}\left(1-\omega_{1}-k_{1}\right)\left\|T_{\omega_{2}} q-T_{1} T_{\omega_{2}} q\right\|^{2} \\
\leq & \|p-q\|^{2}-\omega_{1}\left(1-\omega_{1}-k_{1}\right)\left\|T_{\omega_{2}} q-T_{1} T_{\omega_{2}} q\right\|^{2} .
\end{aligned}
$$

Since $0<k_{1}<\omega_{1}<1 / 2$, we obtain

$$
\left\|T_{\omega_{2}} q-T_{1} T_{\omega_{2}} q\right\|^{2} \leq 0 .
$$


Namely, $T_{\omega_{2}} q=T_{1} T_{\omega_{2}} q$, that is:

$$
T_{\omega_{2}} q \in F_{i x}\left(T_{1}\right)=F_{i x}\left(T_{\omega_{1}}\right), \quad T_{\omega_{2}} q=T_{\omega_{1}} T_{\omega_{2}} q
$$

By induction, we also claim that the Lemma 2.11(b) holds.

Lemma 2.12. Let $K$ be a closed convex subset of a real Hilbert space $H$, given $x \in H$ and $y \in K$. Then $y=P_{K} x$ if and only if there holds the inequality

$$
\langle x-y, y-z\rangle \geq 0, \quad \forall z \in K
$$

\section{Cyclic Algorithm}

In this section, we are concerned with the problem of finding a point $p$ such that

$$
p \in \bigcap_{i=1}^{N} F_{i x}\left(T_{\omega_{i}}\right)=\bigcap_{i=1}^{N} F_{i x}\left(T_{i}\right), \quad N \geq 1,
$$

where $T_{\omega_{i}}=\left(1-\omega_{i}\right) I+\omega_{i} T_{i},\left\{\omega_{i}\right\}_{i=1}^{N} \in(0,1 / 2]$ and $\left\{T_{i}\right\}_{i=1}^{N}$ are $k_{i}$-strictly pseudononspreading mappings with $k_{i} \in\left[0, \omega_{i}\right),(i=1,2, \ldots, N)$, defined on a closed convex subset $C$ in Hilbert space $H$. Here $F_{i x}\left(T_{\omega_{i}}\right)=\left\{q \in C: T_{\omega_{i}} q=q\right\}$ is the set of fixed points of $T_{i}, 1 \leq i \leq N$.

Let $H$ be a real Hilbert space, and let $B: H \rightarrow H$ be $\eta$-strongly monotone and $\rho$ Lipschitzian on $H$ with $\rho>0, \eta>0$. Let $0<\mu<2 \eta / \rho^{2}, 0<\gamma<\mu\left(\eta-\left(\mu \rho^{2} / 2\right)\right) / L=\tau / L$. Let $N$ be a positive integer, and let $T_{i}: H \rightarrow H$ be a $k_{i}$-strictly pseudononspreading mapping for some $k_{i} \in[0,1),(i=1,2, \ldots, N)$, such that $\bigcap_{i=1}^{N} F_{i x}\left(T_{i}\right) \neq \emptyset$. We consider the problem of finding $p \in \bigcap_{i=1}^{N} F_{i x}\left(T_{i}\right)$ such that

$$
\langle(\gamma f-\mu B) p, v-p\rangle \leq 0, \quad \forall v \in \bigcap_{i=1}^{N} F_{i x}\left(T_{i}\right) .
$$

Since $\bigcap_{i=1}^{N} F_{i x}\left(T_{i}\right)$ is a nonempty closed convex subset of $H$, VI (3.2) has a unique solution. The variational inequality has been extensively studied in literature; see, for example, [12-16].

Remark 3.1. Let $H$ be a real Hilbert space. Let $B$ be a $\rho$-Lipschitzian and $\eta$-strongly monotone operator on $H$ with $\rho>0, \eta>0$. Leting $0<\mu<2 \eta / \rho^{2}$ and leting $S=(I-t \mu B)$ and $\mu\left(\eta-\left(\mu \rho^{2} / 2\right)\right)=\tau$, then for $\forall x, y \in H$ and $t \in(0, \min \{1,1 / \tau\}), S$ is a contraction.

Proof. Consider

$$
\begin{aligned}
\|S x-S y\|^{2} & =\|(I-t \mu B) x-(I-t \mu B) y\|^{2} \\
& =\langle(I-t \mu B) x-(I-t \mu B) y,(I-t \mu B) x-(I-t \mu B) y\rangle \\
& =\|x-y\|^{2}+t^{2} \mu^{2}\|B x-B y\|^{2}-2 t \mu\langle x-y, B x-B y\rangle
\end{aligned}
$$




$$
\begin{aligned}
& \leq\|x-y\|^{2}+t^{2} \mu^{2} \rho^{2}\|x-y\|^{2}-2 t \mu \eta\|x-y\|^{2} \\
& \leq\left[1-2 t \mu\left(\eta-\frac{\mu \rho^{2}}{2}\right)\right]\|x-y\|^{2} \\
& =(1-2 t \tau)\|x-y\|^{2} \\
& \leq(1-t \tau)^{2}\|x-y\|^{2} .
\end{aligned}
$$

It follows that

$$
\|S x-S y\| \leq(1-t \tau)\|x-y\| .
$$

So $S$ is a contraction.

Next, we consider the cyclic algorithm (1.15), respectively, for solving the variational inequality over the set of the common fixed points of finite strictly pseudononspreading mappings.

Lemma 3.2. Assume that $\left\{x_{n}\right\}$ is defined by (1.15); if $p$ is solution of (3.2) with $T: H \rightarrow H$ being strictly pseudononspreading mapping and demiclosed and $\left\{y_{n}\right\} \subset H$ is a bounded sequence such that $\left\|T y_{n}-y_{n}\right\| \rightarrow 0$, then

$$
\liminf _{n \rightarrow \infty}\left\langle(\gamma f-\mu B) p, y_{n}-p\right\rangle \leq 0
$$

Proof. By $\left\|T y_{n}-y_{n}\right\| \rightarrow 0$ and $T: H \rightarrow H$ demi-closed, we know that any weak cluster point of $\left\{y_{n}\right\}$ belongs to $\bigcap_{i=1}^{N} F_{i x}\left(T_{i}\right)$. Furthermore, we can also obtain that there exists $\bar{y}$ and a subsequence $\left\{y_{n_{j}}\right\}$ of $\left\{y_{n}\right\}$ such that $y_{n_{j}}-\bar{y}$ as $j \rightarrow \infty$ (hence $\bar{y} \in \bigcap_{i=1}^{N} F_{i x}\left(T_{i}\right)$ ) and

$$
\liminf _{n \rightarrow \infty}\left\langle(\gamma f-\mu B) p, y_{n}-p\right\rangle=\lim _{j \rightarrow \infty}\left\langle(\gamma f-\mu B) p, y_{n_{j}}-p\right\rangle
$$

From (3.2), we can derive that

$$
\liminf _{n \rightarrow \infty}\left\langle(\gamma f-\mu B) p, y_{n}-p\right\rangle=\langle(\gamma f-\mu B) p, \bar{y}-p\rangle \leq 0
$$

It is the desired result. In addition, the variational inequality (3.7) can be written as

$$
\langle(I-\mu B+\gamma f) p-p, \bar{y}-p\rangle \leq 0, \quad \bar{y} \in \bigcap_{i=1}^{N} F_{i x}\left(T_{i}\right) .
$$


So, by Lemma 2.12 , it is equivalent to the fixed point equation

$$
P_{\bigcap_{i=1}^{N} F_{i x}\left(T_{i}\right)}(I-\mu B+\gamma f) p=p
$$

Theorem 3.3. Let $C$ be a nonempty closed convex subset of $H$ and for $1 \leq i \leq N$. Let $T_{i}: H \rightarrow H$ be $k_{i}$-strictly pseudononspreading mappings for some $k_{i} \in\left[0, \omega_{i}\right), \omega_{i} \in(0,1 / 2),(i=1,2, \ldots, N)$, and $k=\max \left\{k_{i}: 1 \leq i \leq N\right\}$. Let $f$ be L-Lipschitz mapping on $H$ with coefficient $L>0$, and let $B: H \rightarrow H$ be $\eta$-strongly monotone and $\rho$-Lipschitzian on $H$ with $\rho>0, \eta>0$. Let $\left\{\alpha_{n}\right\}$ being $a$ sequence in $(0, \min \{1,1 / \tau\})$ satisfying the following conditions:

(c1) $\lim _{n \rightarrow \infty} \alpha_{n}=0$,

(c2) $\sum_{n=0}^{\infty} \alpha_{n}=\infty$.

Given $x_{0} \in C$, let $\left\{x_{n}\right\}_{n=1}^{\infty}$ be the sequence generated by the cyclic algorithm (1.15). Then $\left\{x_{n}\right\}$ converges strongly to the unique element $p$ in $\bigcap_{i=1}^{N} F_{i x}\left(T_{i}\right)$ verifying

$$
p=P_{\bigcap_{i=1}^{N} F_{i x}\left(T_{i}\right)}(I-\mu B+\gamma f) p \text {, }
$$

which equivalently solves the variational inequality problem (3.2).

Proof. Take a $p \in \bigcap_{i=1}^{N} F_{i x}\left(T_{i}\right)$. Let $T_{\omega} x=(1-\omega) x+\omega T x$ and $0<k<\omega<1 / 2$. Then $\forall x, y \in C$, we have

$$
\begin{aligned}
\left\|T_{\omega} x-T_{\omega} y\right\|^{2}= & \omega\|x-y\|^{2}+(1-\omega)\|T x-T y\|^{2}-\omega(1-\omega)\|x-T x-(y-T y)\|^{2} \\
\leq & \omega\|x-y\|^{2}+(1-\omega)\left[\|x-y\|^{2}+k\|x-T x-(y-T y)\|^{2}+2\langle x-T x, y-T y\rangle\right] \\
& -\omega(1-\omega)\|x-T x-(y-T y)\|^{2} \\
= & \|x-y\|^{2}+2(1-\omega)\langle x-T x, y-T y\rangle-(1-\omega)(\omega-k)\|x-T x-(y-T y)\|^{2} \\
\leq & \|x-y\|^{2}+2(1-\omega)\langle x-T x, y-T y\rangle \\
= & \|x-y\|^{2}+\frac{2(1-\omega)}{\omega^{2}}\left\langle x-T_{\omega} x, y-T_{\omega} y\right\rangle .
\end{aligned}
$$

From $p \in F_{i x}(T)$ and (3.11), we also have

$$
\left\|T_{\omega} x_{n}-p\right\| \leq\left\|x_{n}-p\right\| .
$$

Using (1.15) and (3.12), we obtain

$$
\begin{aligned}
\left\|x_{n+1}-p\right\| & =\left\|\alpha_{n} \gamma\left(f\left(x_{n}\right)-f(p)\right)+\alpha_{n}(\gamma f(p)-p)+\left(I-\mu \alpha_{n} B\right)\left(T_{\omega_{[n]}} x_{n}-p\right)\right\| \\
& \leq \alpha_{n} \gamma\left\|f\left(x_{n}\right)-f(p)\right\|+\alpha_{n}\|\gamma f(p)-p\|+\left(1-\alpha_{n} \tau\right)\left\|x_{n}-p\right\|,
\end{aligned}
$$


which combined with (3.12) and $\left\|f\left(x_{n}\right)-f(p)\right\| \leq L\left\|x_{n}-p\right\|$ amounts to

$$
\left\|x_{n+1}-p\right\| \leq\left(1-\alpha_{n}(\tau-\gamma L)\right)\left\|x_{n}-p\right\|+\alpha_{n}\|\gamma f(p)-p\|
$$

Putting $M_{1}=\max \left\{\left\|x_{0}-p\right\|,\|\gamma f(p)-p\|\right\}$, we clearly obtain $\left\|x_{n}-p\right\| \leq M_{1}$. Hence $\left\{x_{n}\right\}$ is bounded. We can also prove that the sequences $\left\{f\left(x_{n}\right)\right\}$ and $\left\{T_{\omega_{[n]}} x_{n}\right\}$ are all bounded.

From (1.15) we obtain that

$$
x_{n+1}-x_{n}+\alpha_{n}\left(\mu B x_{n}-\gamma f\left(x_{n}\right)\right)=\left(I-\mu \alpha_{n} B\right)\left(T_{\omega_{[n]}} x_{n}-x_{n}\right),
$$

hence

$$
\begin{aligned}
& \left\langle x_{n+1}-x_{n}+\alpha_{n}\left(\mu B x_{n}-\gamma f\left(x_{n}\right)\right), x_{n}-p\right\rangle \\
= & \left(1-\alpha_{n}\right)\left\langle T_{\omega_{[n]}} x_{n}-x_{n}, x_{n}-p\right\rangle+\alpha_{n}\left\langle(I-\mu B)\left(T_{\omega_{[n]}} x_{n}-x_{n}\right), x_{n}-p\right\rangle \\
= & \left(1-\alpha_{n}\right)\left\langle T_{\omega_{[n]}} x_{n}-x_{n}, x_{n}-p\right\rangle+\alpha_{n}\left\|(I-\mu B)\left(T_{\omega_{[n]}} x_{n}-x_{n}\right)\right\|\left\|x_{n}-p\right\| \\
= & \left(1-\alpha_{n}\right)\left\langle T_{\omega_{[n]}} x_{n}-x_{n}, x_{n}-p\right\rangle+\alpha_{n}(1-\tau)\left\|T_{\omega_{[n]}} x_{n}-x_{n}\right\|\left\|x_{n}-p\right\| \\
= & \left(1-\alpha_{n}\right)\left\langle T_{\omega_{[n]}} x_{n}-x_{n}, x_{n}-p\right\rangle+\omega_{[n]} \alpha_{n}(1-\tau)\left\|T_{[n]} x_{n}-x_{n}\right\|\left\|x_{n}-p\right\| .
\end{aligned}
$$

Moreover, by $p \in \bigcap_{i=1}^{N} F_{i x}\left(T_{i}\right)$ and using Remark 2.5, we obtain

$$
\left\langle x_{n}-T_{\omega_{[n]}} x_{n}, x_{n}-p\right\rangle \geq \frac{1}{2} \omega_{[n]}\left(1-k_{[n]}\right)\left\|x_{n}-T_{[n]} x_{n}\right\|^{2}
$$

which combined with (3.16) entails

$$
\begin{aligned}
\left\langle x_{n+1}-x_{n}+\alpha_{n}(\mu B-\gamma f) x_{n}, x_{n}-p\right\rangle \leq & -\frac{1}{2} \omega_{[n]}\left(1-k_{[n]}\right)\left(1-\alpha_{n}\right)\left\|x_{n}-T_{[n]} x_{n}\right\|^{2} \\
& +\omega_{[n]} \alpha_{n}(1-\tau)\left\|T_{[n]} x_{n}-x_{n}\right\|\left\|x_{n}-p\right\|
\end{aligned}
$$

or equivalently

$$
\begin{gathered}
-\left\langle x_{n}-x_{n+1}, x_{n}-p\right\rangle \\
\leq-\alpha_{n}\left\langle(\mu B-\gamma f) x_{n}, x_{n}-p\right\rangle-\frac{1}{2} \omega_{[n]}\left(1-k_{[n]}\right)\left(1-\alpha_{n}\right)\left\|x_{n}-T_{[n]} x_{n}\right\|^{2} \\
+\omega_{[n]} \alpha_{n}(1-\tau)\left\|T_{[n]} x_{n}-x_{n}\right\|\left\|x_{n}-p\right\| .
\end{gathered}
$$

Furthermore, using the following classical equality

$$
\langle u, v\rangle=\frac{1}{2}\|u\|^{2}-\frac{1}{2}\|u-v\|^{2}+\frac{1}{2}\|v\|^{2}, \quad \forall u, v \in C
$$


and setting $\tau_{n}=1 / 2\left\|x_{n}-p\right\|^{2}$, we have

$$
\left\langle x_{n}-x_{n+1}, x_{n}-p\right\rangle=\tau_{n}-\tau_{n+1}+\frac{1}{2}\left\|x_{n}-x_{n+1}\right\|^{2} .
$$

So (3.19) can be equivalently rewritten as

$$
\begin{aligned}
\tau_{n+1}-\tau_{n}-\frac{1}{2}\left\|x_{n}-x_{n+1}\right\|^{2} \leq & -\alpha_{n}\left\langle(\mu B-\gamma f) x_{n}, x_{n}-p\right\rangle \\
& -\frac{1}{2} \omega_{[n]}\left(1-k_{[n]}\right)\left(1-\alpha_{n}\right)\left\|x_{n}-T_{[n]} x_{n}\right\|^{2} \\
& +\omega_{[n]} \alpha_{n}(1-\tau)\left\|T_{[n]} x_{n}-x_{n}\right\|\left\|x_{n}-p\right\| .
\end{aligned}
$$

Now using (3.15) again, we have

$$
\left\|x_{n+1}-x_{n}\right\|^{2}=\left\|\alpha_{n}\left(\gamma f\left(x_{n}\right)-\mu B x_{n}\right)+\left(I-\mu \alpha_{n} B\right)\left(T_{\omega_{[n]}} x_{n}-x_{n}\right)\right\|^{2} .
$$

Since $B: H \rightarrow H$ is $\eta$-strongly monotone and $k$-Lipschitzian on $H$, hence it is a classical matter to see that

$$
\left\|x_{n+1}-x_{n}\right\|^{2} \leq 2 \alpha_{n}^{2}\left\|\gamma f\left(x_{n}\right)-\mu B x_{n}\right\|^{2}+2\left(1-\alpha_{n} \tau\right)^{2}\left\|T_{\omega_{[n]}} x_{n}-x_{n}\right\|^{2},
$$

which by $\left\|T_{\omega_{[n]}} x_{n}-x_{n}\right\|=\omega_{[n]}\left\|x_{n}-T_{[n]} x_{n}\right\|$ and $\left(1-\alpha_{n} \tau\right)^{2} \leq\left(1-\alpha_{n} \tau\right)$ yields

$$
\frac{1}{2}\left\|x_{n+1}-x_{n}\right\|^{2} \leq \alpha_{n}^{2}\left\|\gamma f\left(x_{n}\right)-\mu B x_{n}\right\|^{2}+\left(1-\alpha_{n} \tau\right) \omega_{[n]}^{2}\left\|x_{n}-T_{[n]} x_{n}\right\|^{2} .
$$

Then from (3.22) and (3.25), we have

$$
\begin{gathered}
\tau_{n+1}-\tau_{n}+\omega_{[n]}\left(\frac{1}{2}\left(1-k_{[n]}\right)\left(1-\alpha_{n}\right)-\omega_{[n]}\left(1-\alpha_{n} \tau\right)\right)\left\|x_{n}-T_{[n]} x_{n}\right\|^{2} \\
\leq \alpha_{n}\left(\alpha_{n}\left\|\gamma f\left(x_{n}\right)-\mu B x_{n}\right\|^{2}-\left\langle(\mu B-\gamma f) x_{n}, x_{n}-p\right\rangle+\omega_{[n]}(1-\tau)\left\|T_{[n]} x_{n}-x_{n}\right\|\left\|x_{n}-p\right\|\right) .
\end{gathered}
$$

The rest of the proof will be divided into two parts.

Case 1. Suppose that there exists $n_{0}$ such that $\left\{\tau_{n}\right\}_{n \geq n_{0}}$ is nonincreasing. In this situation, $\left\{\tau_{n}\right\}$ is then convergent because it is also nonnegative (hence it is bounded from below), so that $\lim _{n \rightarrow \infty}\left(\tau_{n+1}-\tau_{n}\right)=0$; hence, in light of (3.26) together with $\lim _{n \rightarrow \infty} \alpha_{n}=0$ and the boundedness of $\left\{x_{n}\right\}$, we obtain

$$
\lim _{n \rightarrow \infty}\left\|x_{n}-T_{[n]} x_{n}\right\|=0
$$


It also follows from (3.26) that

$$
\begin{gathered}
\tau_{n}-\tau_{n+1} \geq \alpha_{n}\left(-\alpha_{n}\left\|\gamma f\left(x_{n}\right)-\mu B x_{n}\right\|^{2}+\left\langle(\mu B-\gamma f) x_{n}, x_{n}-p\right\rangle\right. \\
\left.+\omega_{[n]}(1-\tau)\left\|T_{[n]} x_{n}-x_{n}\right\|\left\|x_{n}-p\right\|\right) .
\end{gathered}
$$

Then, by $\sum_{n=0}^{\infty} \alpha_{n}=\infty$, we obviously deduce that

$$
\begin{gathered}
\liminf _{n \rightarrow \infty} \alpha_{n}\left(-\alpha_{n}\left\|\gamma f\left(x_{n}\right)-\mu B x_{n}\right\|^{2}+\left\langle(\mu B-\gamma f) x_{n}, x_{n}-p\right\rangle\right. \\
\left.+\omega_{[n]}(1-\tau)\left\|T_{[n]} x_{n}-x_{n}\right\|\left\|x_{n}-p\right\|\right) \leq 0,
\end{gathered}
$$

or equivalently (as $\alpha_{n}\left\|\gamma f\left(x_{n}\right)-\mu B x_{n}\right\|^{2} \rightarrow 0$ )

$$
\liminf _{n \rightarrow \infty}\left\langle(\mu B-\gamma f) x_{n}, x_{n}-p\right\rangle \leq 0
$$

Moreover, by Remark 1.2, we have

$$
2(\mu \eta-\gamma L) \tau_{n}+\left\langle(\mu B-\gamma f) p, x_{n}-p\right\rangle \leq\left\langle(\mu B-\gamma f) x_{n}, x_{n}-p\right\rangle,
$$

which by (3.30) entails

$$
\liminf _{n \rightarrow \infty}\left\langle 2(\mu \eta-\gamma L) \tau_{n}+(\mu B-\gamma f) p, x_{n}-p\right\rangle \leq 0,
$$

hence, recalling that $\lim _{n \rightarrow \infty} \tau_{n}$ exists, we equivalently obtain

$$
2(\mu \eta-\gamma L) \lim _{n \rightarrow \infty} \tau_{n}+\liminf _{n \rightarrow \infty}\left\langle(\mu B-\gamma f) p, x_{n}-p\right\rangle \leq 0,
$$

namely

$$
2(\mu \eta-\gamma L) \lim _{n \rightarrow \infty} \tau_{n} \leq-\liminf _{n \rightarrow \infty}\left\langle(\mu B-\gamma f) p, x_{n}-p\right\rangle .
$$

From (3.27) and Lemma 3.2, we obtain

$$
\liminf _{n \rightarrow \infty}\left\langle(\mu B-\gamma f) p, x_{n}-p\right\rangle \geq 0
$$

which yields $\lim _{n \rightarrow \infty} \tau_{n}=0$, so that $\left\{x_{n}\right\}$ converges strongly to $p$. 
Journal of Applied Mathematics

Case 2. Suppose there exists a subsequence $\left\{\tau_{n_{k}}\right\}_{k \geq 0}$ of $\left\{\tau_{n}\right\}_{n \geq 0}$ such that $\tau_{n_{k}} \leq \tau_{n_{k+1}}$ for all $k \geq 0$. In this situation, we consider the sequence of indices $\{\delta(n)\}$ as defined in Lemma 2.8. It follows that $\tau_{\delta(n+1)}-\tau_{\delta(n)}>0$, which by (3.26) amounts to

$$
\begin{gathered}
\omega_{[n]}\left(\frac{1}{2}\left(1-k_{[n]}\right)\left(1-\alpha_{\delta(n)}\right)-\omega_{[n]}\left(1-\alpha_{\delta(n)} \tau\right)\right)\left\|x_{\delta(n)}-T_{[n]} x_{\delta(n)}\right\|^{2} \\
<\alpha_{\delta(n)}\left(\alpha_{\delta(n)}\left\|\gamma f\left(x_{\delta(n)}\right)-\mu B x_{\delta(n)}\right\|^{2}-\left\langle(\mu B-\gamma f) x_{\delta(n)}, x_{\delta(n)}-p\right\rangle\right. \\
\left.+\omega_{[n]}(1-\tau)\left\|T_{[n]} x_{\delta(n)}-x_{\delta(n)}\right\|\left\|x_{\delta(n)}-p\right\|\right) .
\end{gathered}
$$

By the boundedness of $\left\{x_{n}\right\}$ and $\lim _{n \rightarrow \infty} \alpha_{n}=0$, we immediately obtain

$$
\lim _{n \rightarrow \infty}\left\|x_{\delta(n)}-T_{[n]} x_{\delta(n)}\right\|=0 .
$$

Using (1.15), we have

$$
\begin{aligned}
\left\|x_{\delta(n)+1}-x_{\delta(n)}\right\| & \leq \alpha_{\delta(n)}\left\|\gamma f\left(x_{\delta(n)}\right)-\mu B x_{\delta(n)}\right\|+\left(1-\alpha_{\delta(n)} \tau\right)\left\|T_{\omega_{[n]}} x_{\delta(n)}-x_{\delta(n)}\right\| \\
& \leq \alpha_{\delta(n)}\left\|\gamma f\left(x_{\delta(n)}\right)-\mu B x_{\delta(n)}\right\|+\omega_{[n]}\left(1-\alpha_{\delta(n)} \tau\right)\left\|T_{[n]} x_{\delta(n)}-x_{\delta(n)}\right\|,
\end{aligned}
$$

which together with (3.37) and $\lim _{n \rightarrow \infty} \alpha_{n}=0$ yields

$$
\lim _{n \rightarrow \infty}\left\|x_{\delta(n)+1}-x_{\delta(n)}\right\|=0
$$

Now by (3.36) we clearly have

$$
\begin{gathered}
\alpha_{\delta(n)}\left\|\gamma f\left(x_{\delta(n)}\right)-\mu B x_{\delta(n)}\right\|^{2}+\omega_{[n]}(1-\tau)\left\|T_{[n]} x_{\delta(n)}-x_{\delta(n)}\right\|\left\|x_{\delta(n)}-p\right\| \\
\geq\left\langle(\mu B-\gamma f) x_{\delta(n)}, x_{\delta(n)}-p\right\rangle,
\end{gathered}
$$

which in the light of (3.31) yields

$$
\begin{gathered}
2(\mu \eta-\gamma L) \tau_{\delta(n)}+\left\langle(\mu B-\gamma f) p, x_{\delta(n)}-p\right\rangle \\
\leq \alpha_{\delta(n)}\left\|\gamma f\left(x_{\delta(n)}\right)-\mu B x_{\delta(n)}\right\|^{2}+\omega_{[n]}(1-\tau)\left\|T_{[n]} x_{\delta(n)}-x_{[n]}\right\|\left\|x_{\delta(n)}-p\right\|,
\end{gathered}
$$

hence $\left(\operatorname{as} \lim _{n \rightarrow \infty} \alpha_{\delta(n)}\left\|\gamma f\left(x_{\delta(n)}\right)-\mu B x_{\delta(n)}\right\|^{2}=0\right.$ and (3.37)) it follows that

$$
2(\mu \eta-\gamma L) \limsup _{n \rightarrow \infty} \tau_{\delta(n)} \leq-\liminf _{n \rightarrow \infty}\left\langle(\mu B-\gamma f) p, x_{\delta(n)}-p\right\rangle .
$$

From (3.37) and Lemma 3.2, we obtain

$$
\lim _{n \rightarrow \infty}\left\langle(\mu B-\gamma f) p, x_{\delta(n)}-p\right\rangle \geq 0,
$$


which by (3.42) yields $\limsup _{n \rightarrow \infty} \tau_{\delta(n)}=0$, so that $\lim _{n \rightarrow \infty} \tau_{\delta(n)}=0$. Combining (3.39), we have $\lim _{n \rightarrow \infty} \tau_{\delta(n)+1}=0$. Then, recalling that $\tau_{n}<\tau_{\delta(n)+1}$ (by Lemma 2.8), we get $\lim _{n \rightarrow \infty} \tau_{n}=0$, so that $x_{n} \rightarrow p$ strongly.

Taking $k_{i}=0$, we know that $k_{i}$-strictly pseudononspreading mapping is nonspreading mapping and $i=n(\bmod N), 0 \leq i \leq N-1$. According to the proof Theorem 3.3, we obtain the following corollary.

Corollary 3.4. Let $C$ be a nonempty closed convex subset of $H$. Let $T_{i}: C \rightarrow C$ be nonspreading mappings and $\omega_{i} \in(0,1 / 2),(i=1,2, \ldots, N)$. Let $f$ be L-Lipschitz mapping on $H$ with coefficient $L>0$ and let $B: H \rightarrow H$ be $\eta$-strongly monotone and $\rho$-Lipschitzian on $H$ with $\rho>0, \eta>0$. Let $\left\{\alpha_{n}\right\}$ be a sequence in $(0, \min \{1,1 / \tau\})$ satisfying the following conditions:

(c1) $\lim _{n \rightarrow \infty} \alpha_{n}=0$,

(c2) $\sum_{n=0}^{\infty} \alpha_{n}=\infty$.

Given $x_{0} \in C$, let $\left\{x_{n}\right\}_{n=1}^{\infty}$ be the sequence generated by the cyclic algorithm (1.15). Then $\left\{x_{n}\right\}$ converges strongly to the unique element $p$ in $\bigcap_{i=1}^{N} F_{i x}\left(T_{i}\right)$ verifying

$$
p=P_{\bigcap_{i=1}^{N} F_{i x}\left(T_{i}\right)}(I-\mu B+\gamma f) p,
$$

which equivalently solves the variational inequality problem (3.2).

\section{Acknowledgment}

This work is supported in part by China Postdoctoral Science Foundation (Grant no. 20100470783).

\section{References}

[1] F. E. Browder and W. V. Petryshyn, "Construction of fixed points of nonlinear mappings in Hilbert space," Journal of Mathematical Analysis and Applications, vol. 20, pp. 197-228, 1967.

[2] M. O. Osilike and F. O. Isiogugu, "Weak and strong convergence theorems for nonspreading-type mappings in Hilbert spaces," Nonlinear Analysis: Theory, Methods $\mathcal{E}$ Applications, vol. 74, no. 5, pp. 1814-1822, 2011.

[3] S. Iemoto and W. Takahashi, "Approximating common fixed points of nonexpansive mappings and nonspreading mappings in a Hilbert space," Nonlinear Analysis: Theory, Methods E Applications, vol. 71, no. 12, pp. e2082-e2089, 2009.

[4] K. Aoyama, "Halperns iteration for a sequence of quasinonexpansive type mappings," Nonlinear Mathematics for Uncertainty and Its Applications, vol. 100, pp. 387-394, 2011.

[5] K. Aoyama and F. Kohsaka, "Fixed point and mean convergence theorems for a famliy of $\lambda$-hybrid mappings," Journal of Nonlinear Analysis and Optimization, vol. 2, no. 1, pp. 85-92, 2011.

[6] N. Petrot and R. Wangkeeree, "A general iterative scheme for strict pseudononspreading mapping related to optimization problem in Hilbert spaces," Journal of Nonlinear Analysis and Optimization, vol. 2, no. 2, pp. 329-336, 2011.

[7] G. L. Acedo and H.-K. Xu, "Iterative methods for strict pseudo-contractions in Hilbert spaces," Nonlinear Analysis: Theory, Methods \& Applications, vol. 67, no. 7, pp. 2258-2271, 2007.

[8] S. A. Naimpally and K. L. Singh, "Extensions of some fixed point theorems of Rhoades," Journal of Mathematical Analysis and Applications, vol. 96, no. 2, pp. 437-446, 1983.

[9] T. L. Hicks and J. D. Kubicek, "On the Mann iteration process in a Hilbert space," Journal of Mathematical Analysis and Applications, vol. 59, no. 3, pp. 498-504, 1977. 
[10] P.-E. Maingé, "The viscosity approximation process for quasi-nonexpansive mappings in Hilbert spaces," Computers $\mathcal{E}$ Mathematics with Applications, vol. 59, no. 1, pp. 74-79, 2010.

[11] P.-E. Maingé, "Strong convergence of projected subgradient methods for nonsmooth and nonstrictly convex minimization," Set-Valued Analysis, vol. 16, no. 7-8, pp. 899-912, 2008.

[12] S. Plubtieng and R. Punpaeng, "A new iterative method for equilibrium problems and fixed point problems of nonexpansive mappings and monotone mappings," Applied Mathematics and Computation, vol. 197, no. 2, pp. 548-558, 2008.

[13] H.-K. Xu, "Iterative algorithms for nonlinear operators," Journal of the London Mathematical Society Second Series, vol. 66, no. 1, pp. 240-256, 2002.

[14] H.-K. Xu, "Viscosity approximation methods for nonexpansive mappings," Journal of Mathematical Analysis and Applications, vol. 298, no. 1, pp. 279-291, 2004.

[15] H. K. Xu, "An iterative approach to quadratic optimization," Journal of Optimization Theory and Applications, vol. 116, no. 3, pp. 659-678, 2003.

[16] L. C. Zeng, S. Schaible, and J. C. Yao, "Iterative algorithm for generalized set-valued strongly nonlinear mixed variational-like inequalities," Journal of Optimization Theory and Applications, vol. 124, no. 3, pp. 725-738, 2005. 


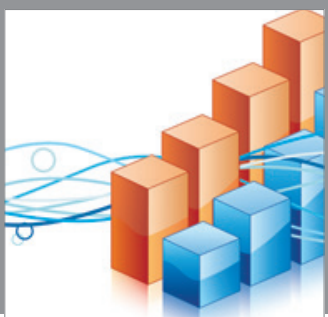

Advances in

Operations Research

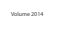

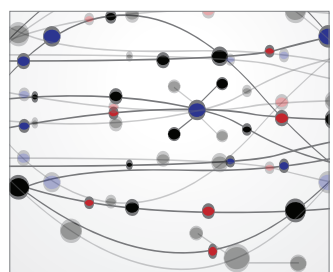

\section{The Scientific} World Journal
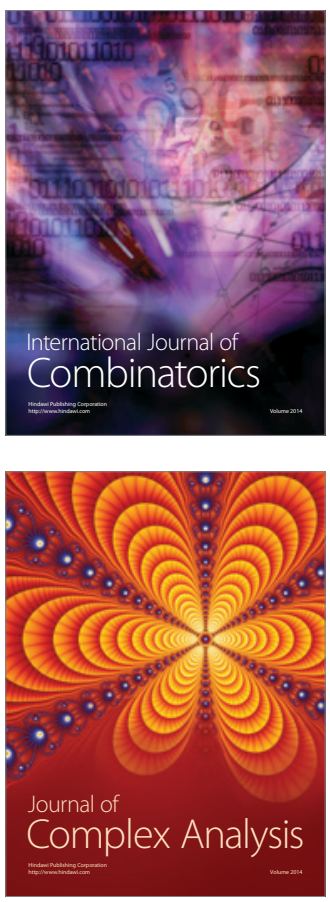

International Journal of

Mathematics and

Mathematical

Sciences
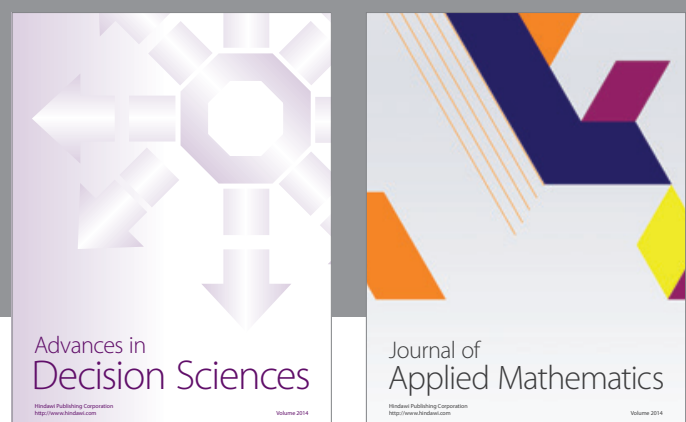

Journal of

Applied Mathematics
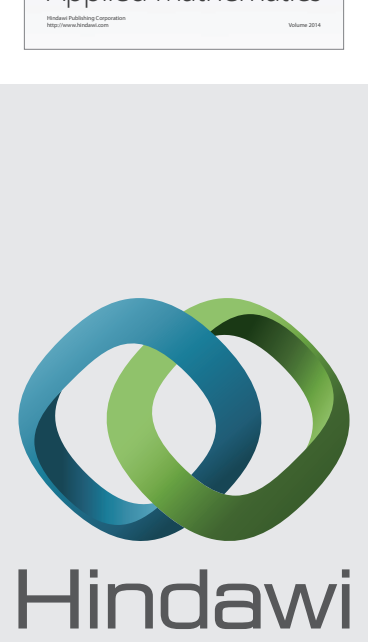

Submit your manuscripts at http://www.hindawi.com
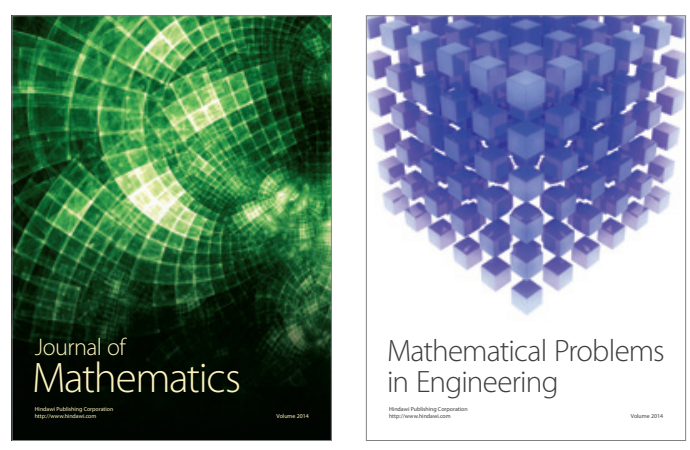

Mathematical Problems in Engineering
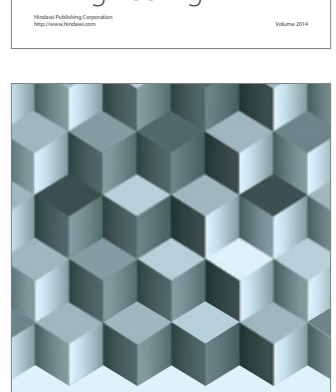

Journal of

Function Spaces
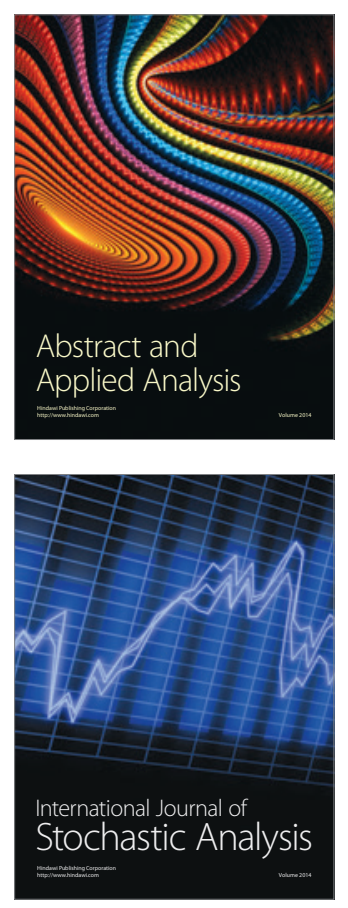

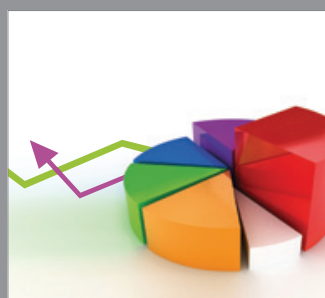

ournal of

Probability and Statistics

Promensencen
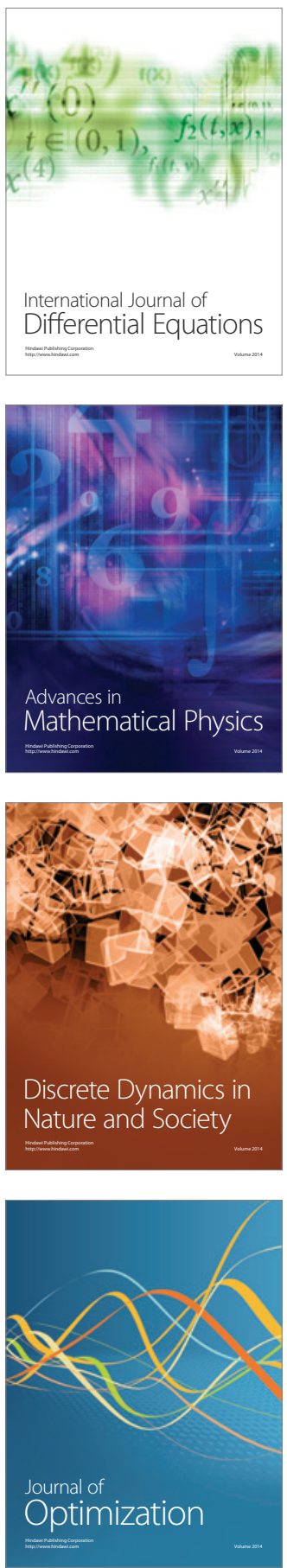